\title{
Neural Crest-Derived Dental Pulp Stem Cells Function as Ectomesenchyme to Support Salivary Gland Tissue Formation
}

\author{
Kajohnkiart Janebodin ${ }^{1,3}$ and Morayma Reyes ${ }^{1,2 *}$
}

${ }^{1}$ Department of Oral Health Sciences, School of Dentistry, University of Washington, Seattle, Washington, USA

${ }^{2}$ Department of Pathology, School of Medicine, University of Washington, Seattle, Washington, USA

${ }^{3}$ Department of Anatomy, Faculty of Dentistry, Mahidol University, Bangkok, Thailand

\begin{abstract}
Xerostomia, dry mouth due to loss of functional salivary gland, is caused by Sjögren's syndrome, radiotherapy for head and neck cancer, medications and aging, leading to patients' suffer from difficulties in swallowing and speech, as well as oral diseases. Stem cell therapy is considered a potential therapeutic alternative. However, combinatory approaches including not only salivary gland stem cells but also supportive cells and appropriate extracellular matrix are necessary to form a functional salivary gland. Like tooth formation, the development of salivary gland requires epithelium interacting with neural crest-derived mesenchyme. Dental pulp stem cells (DPSC) isolated from murine dental pulp is neural crest-derived. Herein, we used the human salivary gland (HSG) cell line as a model to study the effects of DPSC on salivary gland differentiation. Upon in vitro differentiation on Matrigel, HSG alone and HSG cocultured with Wnt1-Cre/R26R-LacZ derived DPSC (HSG+DPSC) differentiated into acinar-like structures. However, HSG formed more mature (higher expression of LAMP-1 and CD44), larger and increased numbers of acinar structures in HSG+DPSC. In vivo subcutaneous co-transplantation of HSG and DPSC with hyaluronic acid (HA) hydrogel after 2 weeks was evaluated by Q-RT- PCR, morphological and immunohistological assessment. Compared to HSG transplants which only showed undifferentiated tumor-like cells, HSG+DPSC demonstrated (1) higher expression of murine mesenchymal marker Fgf-7 (2) higher expression of mature human salivary gland differentiation marker alphaamylase-1 AMY-1 (3) higher expression of murine endothelial, vWF, neuronal, NF-200, and angiogenic markers, Vegfr-3 and Vegf-C; (4) mucin-secreting acinar- and duct-like structures with abundant blood vessels at the interface with DPSC; and (5) more mature glandular structures double-positive for salivary gland differentiation markers CD44 and LAMP-1. These results indicate that DPSC supported and enhanced HSG differentiation into functional salivary gland tissue. This study illustrates the potential of DPSC as inductive mesenchyme for salivary gland regeneration, repair, and tissue engineering.
\end{abstract}

\section{Introduction}

Diseases such as xerostomia and radiation treatment for head and neck cancer can induce irreparable salivary gland tissue damage [1]. Salivary gland stem cells have been characterized and shown capable to differentiate into all the epithelial components of the salivary gland tissue such as ductal and acinar cells [2]; however, their use for salivary gland tissue regeneration is hampered by the need of other supportive cells, vasculature, innervations and matrix necessary for functional regeneration of the salivary gland [3]. Although the epithelium of the parotid glands is ectodermal-derived whereas the epithelium of the submandibular and sublingual glands is endodermalderived, the salivary gland mesenchyme is neural crest-derived [4,5]. The interaction of epithelium and mesenchyme is essential for the branching morphogenesis of the salivary gland. Importantly, the epithelial-mesenchymal interactions of tooth bud and salivary glands are similar and molecular cues such as secretion of fibroblast growth factors (FGF-10, FGF-7) by the ecto-mesenchyme and expression of FGF receptors (FGFR-1, FGFR-2) by the epithelium are important for both the development of tooth bud and salivary gland [4-10]. Also important morphogens such as Shh, Wnt, fibroblast growth factors seem to play important roles in tooth bud and saliva gland development [11-13]. We have recently characterized neural crest-derived dental pulp stem cells (DPSC) from neonatal mice that can differentiate into neural crest lineages including mesenchyme, Schwann cells, odontoblasts and pericyte-like cells [14]. Due to similarities in tooth bud and salivary gland development, herein we studied the capacity of neural crest-derived DPSC to support and induce salivary epithelium differentiation.
The human salivary gland cell line, HSG, is a neoplastic cell line originally developed from an intercalated duct cell of an irradiated human submandibular salivary gland. The HSG cell line forms primary adenocarcinoma tumors with malignant characteristics when transplanted into nude mice $[15,16]$. Nonetheless, when cultured on Matrigel the HSG cell line rapidly differentiates into mature salivary epithelium [17]. Thus, the HSG cell is a good cell line to study salivary gland tissue differentiation as it is capable of rapid expansion, and differentiation into multiple salivary gland epithelial cell phenotypes including myoepithelial-like, acinar like, keratinocytelike, chondrocyte-like and mucinous-like cells $[18,19]$.

In order to study the inductive effects of mouse DPSC on HSG differentiation we performed in vitro xeno-cultures of mouse DPSC and HSG on Matrigel and we observed larger and increased number of acini in the co-cultures as compared to HSG cultures alone. Although Matrigel is widely used for in vitro culture [17] its immunogenicity limits its in vivo application [20].

*Corresponding author: Morayma Reyes, Department of Pathology, University of Washington, 815 Mercer Street, Room 332, Box 358050, Seattle, WA 98109, USA Tel: +1 206 6165004; Fax: +1 206 8971540; E-mail: morayma@u.washington.edu

Received January 23, 2012; Accepted February 10, 2012; Published February 12,2012

Citation: Janebodin K, Reyes M (2012) Neural Crest-Derived Dental Pulp Stem Cells Function as Ectomesenchyme to Support Salivary Gland Tissue Formation. Dentistry S13:001. doi:10.4172/2161-1122.S13-001

Copyright: (c) 2012 Janebodin K, et al. This is an open-access article distributed under the terms of the Creative Commons Attribution License, which permits unrestricted use, distribution, and reproduction in any medium, provided the original author and source are credited. 
Thus, we sought extracellular matrix components important for salivary gland tissue development. Hyaluronic acid (HA) is one of the major extracellular matrix components of the developing and adult salivary gland [21,22]. HA accounts for $50 \%$ of all the glycosaminoglycans (GAG) in the basal lamina of the developing submandibular salivary gland [22]. In adult salivary gland tissue, HA accounts for $25 \%$ of all the GAGs synthesized by the secretory units (acini and intercalated ducts) [21]. Furthermore, HA is the ligand for CD44 which is highly expressed by DPSC [14]. Acinar cells also express CD44 $[23,24]$. We hypothesized that HA hydrogels will provide a HA rich basement membrane for close epithelial-mesenchymal interaction and will result in induction of differentiated salivary gland 3D structures.

Tissue engineering for salivary gland restoration is limited by availability of stem cells or cell sources capable of providing all the cellular components necessary to generate a functional salivary gland tissue. Furthermore, vascularization and innervation of engineered salivary gland tissue is a long-lasting challenge. Herein, we report that neural crest-derived DPSC co- transplanted with HSG in HA hydrogels, secrete fibroblast growth factors, namely FGF-7, angiogenic factors such as VEGF-C and support HSG differentiation into mature salivary gland tissue in vivo.

\section{Materials and Methods}

\section{HSG and DPSC culture}

HSG cells, derived from a human submandibular salivary gland, were a gift from Dr. Kenneth Izutsu (Department of Oral Health Sciences, University of Washington). The cells were plated in 10,000 cells $/ \mathrm{cm}^{2}$ on plastic tissue culture dishes (BD Biosciences, Franklin Lakes, NJ) and cultured at $37^{\circ} \mathrm{C}$ under $5 \% \mathrm{CO}_{2}$ in growth media containing Dulbecco's modification of eagle's medium (DMEM) with $4.5 \mathrm{~g} / \mathrm{L}$ glucose, L-glutamine and sodium pyruvate (Cellgro, Manassas, VA) supplemented with $10 \%$ heat-inactivated fetal calf serum (FCS) (HyClone, Logan, UT), 100 units $/ \mathrm{ml}$ penicillin with $100 \mu \mathrm{g} / \mathrm{ml}$ streptomycin (Cellgro).

Murine DPSC derived from 5-day to 10-day Wnt1-Cre/R26R-LacZ mice was isolated and cultured as previously described [14]. Briefly, the pulp tissue from molar teeth was gently isolated from tooth crowns and digested with a combination of 1.2 units $/ \mathrm{ml}$ dispase II, $2 \mathrm{mg} / \mathrm{ml}$ collagenase type IV (Worthington, Lakewood, NJ) and $2 \mathrm{mM} \mathrm{CaCl}_{2}$ in phosphate buffer saline (PBS) for 60 minutes at $37^{\circ} \mathrm{C}$. The single cell suspension was plated in 1000 cells $/ \mathrm{cm}^{2}$ on plastic tissue culture dishes (BD Biosciences) and cultured at $37^{\circ} \mathrm{C}$ under $5 \% \mathrm{O}_{2}$ and $5 \% \mathrm{CO}_{2}$ in stem cell media containing 60\% low-glucose DMEM (Gibco BRL), $40 \%$ MCDB-201 (Sigma), 2\% FCS, $1 \mathrm{X}$ insulin transferrin selenium (ITS) (Cellgro), $1 X$ linoleic acid bovine serum albumin (LA-BSA) (Sigma), 10${ }^{9} \mathrm{M}$ dexamethasone (Sigma), $10^{-4} \mathrm{M}$ ascorbic acid 2-phosphate (Sigma), 100 units $/ \mathrm{ml}$ penicillin with $100 \mu \mathrm{g} / \mathrm{ml}$ streptomycin, and $1 \times 10^{3}$ units $/ \mathrm{ml}$ leukemia-inhibitory factor (LIF; ESGRO) (Millipore, Temecula, CA), supplemented with $10 \mathrm{ng} / \mathrm{mL}$ EGF and 10ng/mL PDGF-BB (Sigma). The media were changed every three days and passaged when adherent cells were more than 50\% confluent. Both cells were passaged using $0.25 \%$ trypsin-EDTA (Cellgro) and replated at a 1:4 dilution under the same culture condition for each cell type.

\section{In vitro differentiation of HSG, DPSC alone, or HSG co- cultured with DPSC on Matrigel}

Cells were cultured on either plastic or Matrigel-coated surfaces. Growth factor-reduced Matrigel (BD Biosciences) was thawed on ice and diluted in DMEM at a final concentration of $2 \mathrm{mg} / \mathrm{ml}$. To form three-dimensional matrix in culture dishes, $100 \mu \mathrm{l}$ of Matrigel was added to 48 - well tissue culture plate $\left(0.75 \mathrm{~cm}^{2}\right.$ per well $)$ and incubated at $37^{\circ} \mathrm{C}$ for 1 hour before cell seeding. HSG cells, DPSC alone, or a combination of HSG with DPSC (2.5 x $10^{4}$ cells per each cell type/ $\mathrm{cm}^{2}$ ) were seeded in either non-coated or Matrigel-coated plastic surfaces with $100 \mu$ l of additional growth media. Culture medium was changed every two days. After 4 days, X-gal was stained to distinguish Wnt1-marked DPSC which express LacZ gene from HSG cells. The cells were fixed with $0.2 \%$ gluteraldehyde / $2 \%$ formaldehyde in PBS for $10 \mathrm{~min}$, and washed with three times of PBS. The fixed cells were subsequently incubated in $\mathrm{X}$-gal solution at $37^{\circ} \mathrm{C}$ overnight with light protection before washing with three times of PBS. The stained cells were photographed and analyzed.

\section{In vivo subcutaneous transplantation of HSG alone or HSG co-transplanted with DPSC with HA hydrogels}

In accordance with approved Institutional Animal Care and Use Committee (IACUC) protocols, HSG alone or HSG combined with murine DPSC $\left(1 \times 10^{6}\right.$ cells/cell type) were separately transplanted into 2-month-old male Rag1 null mice (Jackson Laboratory, Bar Harbor, ME, USA) by subcutaneous transplantation with hyaluronic acid (HA) hydrogel (HyStem, Glycosan) ( $\mathrm{n}=3$ mice/group) according to the manufacturer's protocol. $100 \mu$ l of cell suspension was injected ventrally to the submandibular salivary gland without penetrating the gland. After 2 weeks of transplantation, HA plugs were dissected without involving mouse recipients' salivary gland tissues and fixed with $10 \%$ neutral buffered formalin (NBF) (Sigma) for 30 min at $4^{\circ} \mathrm{C}$ with agitation, then washed with three times of PBS at RT with agitation. The fixed transplanted tissues were embedded in paraffin and cut to 8 - $\mu \mathrm{m}$ thick sections. Sections were analyzed by Hematoxylin and Eosin (H\&E), Periodic Acid Schiff (PAS), and immunofluorescence staining.

\section{Quantitative reverse transcriptase polymerase chain reaction (Q-RT-PCR)}

Undifferentiated cultured DPSC and transplanted tissues (HSG alone or HSG co- transplanted with DPSC) were extracted for total RNA by using the RNeasy Mini kit (Qiagen) and TRIzol reagent (Invitrogen) according to the manufacturer's protocol, respectively. Quantity and purity of RNA was determined by $260 / 280 \mathrm{~nm}$ absorbance. First-strand cDNA was synthesized from 1000 ng of RNA using the High Capacity cDNA synthesis kit from Applied Biosystems per manufacturer's protocols using a randomized primer. Q-RT-PCR primers are included in Supplementary Method.

cDNA (20ng) was prepared using the SYBR green PCR master mix from Applied Biosystems. Reactions were processed by the ABI $7900 \mathrm{HT}$ PCR system with the following parameters: $50^{\circ} \mathrm{C} / 2 \mathrm{~min}$ and $95^{\circ} \mathrm{C} / 10 \mathrm{~min}$, followed by 40 cycles of $95^{\circ} \mathrm{C} / 15 \mathrm{~s}$ and $60^{\circ} \mathrm{C} / 1 \mathrm{~min}$. Results were analyzed using SDS 2.2 software and relative expression calculated using the comparative Ct method. Each sample was run in triplicate reactions for each gene. cDNA of undifferentiated HSG cells and mouse salivary gland tissue were used to calibrate samples. 


\section{Immunofluorescence}

Cultured cells were fixed with $4 \%$ formaldehyde/PBS for $5 \mathrm{~min}$, washed with $1 \%$ BSA in $0.1 \%$ Triton-X 100/PBS, stained with rat-antimouse/human LAMP-1 monoclonal antibody (whole supernatant, Developmental Hybridoma Bank) overnight at $4^{\circ} \mathrm{C}$ following three times of washing. Goat-derived Alexa 488-conjugated secondary antibody (1:800, Invitrogen) was incubated for $1 \mathrm{~h}$ and washed three times. The cells were double-stained with anti-human CD44 monoclonal antibody directly conjugated with PE (CD44-PE, 1:100, eBioscience) for $1 \mathrm{~h}$ at RT, and then washed three times.

Transplanted tissue sections were deparaffinized, rehydrated, and permeabilized with $1 \%$ bovine serum albumin (BSA) in $0.1 \%$ Triton $\mathrm{X}-100 / \mathrm{PBS}$. Then sections were blocked with $10 \%$ normal goat serum for $1 \mathrm{~h}$ at RT, and incubated with primary antibody which is ratanti- mouse/human LAMP-1 or rabbit-anti-mouse vWF polyclonal antibody (1:400, Dako) overnight at $4^{\circ} \mathrm{C}$ following three times of washing. The stained sections were subsequently incubated with goatderived Alexa 488-conjugated secondary antibody (1:800) for $1 \mathrm{~h}$ at $\mathrm{RT}$, and washed three times. Then the sections were incubated by the second set of primary antibody which is anti-human-CD44-PE or anti-mouse-SMA monoclonal antibody directly conjugated with Cy3 (1:400, Sigma) for $1 \mathrm{~h}$ at RT before washing three times. Cells were stained with 4', 6-diamine-2- phenylindol (DAPI, 1:1000) to visualize the nuclei. All antibodies were diluted in $1 \%$ BSA in $0.1 \%$ Triton-X 100/PBS. All immunofluorescence images described in this manuscript was detected using a Zeiss Axiovert 200 fluorescent microscope (Thornwood, NY). Photographs were taken with an onboard

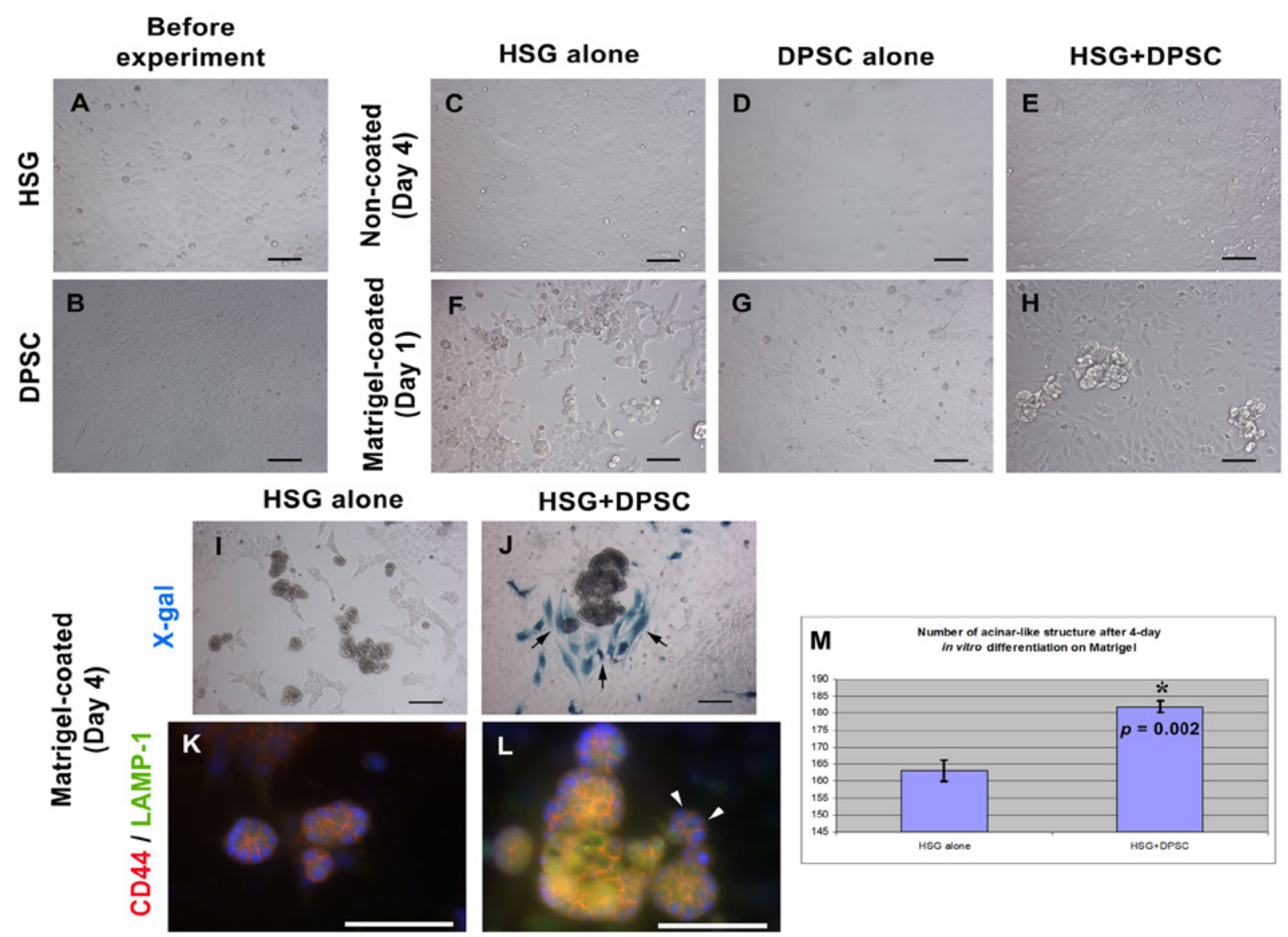

Figure 1: Human Salivary Gland (HSG) Cells formed more mature and increased number of acinar-like structures in the presence of Dental Pulp Stem Cells (DPSC). (A and B) Undifferentiated HSG cells and DPSC cultured on plastic surfaces before starting experiment showed polyhedral- and spindle- shaped, respectively. (C-E) HSG, DPSC alone, and HSG co-cultured with DPSC (HSG+DPSC) cultured on non-coated plastic surfaces proliferated and formed a confluent monolayer. (F-H) HSG, DPSC alone, and HSG+DPSC cultured on Matrigel-coated surface for 1 day. (F) HSG started to form small acinar-like structures. (G) DPSC survived, proliferated, and formed a monolayer, but not gave rise to any acinar-like structures. $(H)$ Large HSG-derived acinar-like structures were found in HSG+DPSC group. (I and J) X-gal showed the positive staining of Wnt1-Cre/R26R-LacZ derived DPSC due to the expression of $\beta$-galactosidase ( $\beta$-gal), but not HSG. (I) HSG-derived acinar-like structures increased in size after 4 days cultured on Matrigel. (J) HSG co-cultured with Wnt-1/ $\beta$-gal+ DPSC (X-gal+ cells shown in blue) formed larger acinar-like structures which closely contacted to DPSC (arrows). ( $\mathrm{K}$ and $\mathrm{L}$ ) The acinar-like structures in both HSG and HSG+DPSC were positively stained for CD44 and LAMP-1. (L) HSG co-cultured with DPSC formed more mature due to higher expression of LAMP-1 and CD44. The ductlike structure was also seen in this group (arrowheads). (M) The bar graph shows that the number of acinar structures increased in HSG+DPSC (182 \pm 0.68 acini) when compared with HSG alone (163 \pm 1.32 acini). The number of acini in both groups were counted from 6 wells $(n=6)$ are statistically significant. Student's t- test calculated * $p \leq 0.05$. Error bars represent \pm SEM. Scale bars indicate $100 \mu \mathrm{m}$. 
monochrome AxioCam MRm camera and colored using Adobe Photoshop (San Jose, CA). Background was reduced using brightness and contrast adjustments, and color balance was performed to enhance colors. All the modifications were applied to the whole image using Adobe Photoshop.

\section{Periodic Acid Schiff (PAS) staining}

Transplanted tissue sections were dewaxed and rehydrated. The sections were stained in $1 \%$ Periodic Acid solution (Sigma) for 10min at RT with agitation before rinsing with deionized water. Then the tissues were incubated in Schiff reagent (Sigma) for $5 \mathrm{~min}$ at RT with agitation before washing three times with deionized water. The stained tissues were dehydrated in a series of alcohol solutions, and cleared with xylene before mounting.

\section{Statistical analysis}

The number of acini formed in the in vitro experiment and the number of blood vessels in the transplanted tissues was quantified using an image analysis program, ImageJ v1.43u (Wayne Rasband, NIH; http://rsb.info.nih.gov/ij). The number of acinar-like structure was counted from 6 wells in each experimental group. The percentage of blood vessel per area was determined upon examination of 10 areas in each experimental group. Data were represented as means \pm the standard error of the mean (SEM) of results from three separate experiments. The data were analyzed by Student's t-test where p-value
$<0.05$ represented significant differences between HSG alone and HSG co-cultured or co-transplanted with DPSC.

\section{Results}

HSG co-cultured with DPSC formed more mature and increased number of acinar-like structures

HSG and DPSC cultured separately on plastic surfaces showed their different cell morphology; the former were polyhedral-shaped epithelial cells whereas the latter were spindle- shaped fibroblasts (Figures 1A and B). As expected, HSG, DPSC alone, and HSG cocultured with DPSC (HSG+DPSC) grown on non-coated surfaces proliferated, but only formed a confluent monolayer (Figures 1C-E).

After cultured separately on Matrigel-coated plastic surfaces for 4 days, HSG underwent dramatically morphological changes in both HSG alone and HSG+DPSC. As previously reported [17], the salivary gland cells initiated to form acinar-like structures after day 1 in Matrigel culture; however, the acinar-like structures found in HSG+DPSC were larger and more numerous than those in HSG alone (Figures 1F and $\mathrm{H})$. The acinar-like structures in HSG alone and HSG+DPSC gradually increased in size (Figures 1I and J). DPSC which were cultured alone on Matrigel did not change their cell morphology (Figure 1G). HSG cultured alone on Matrigel for 4 days showed small acinar-like phenotypes, represented by spherical structures with polarized nuclei and lumen formation (Figure $1 \mathrm{~K}$ ) $[6,25]$. In contrast, HSG in the co-
A

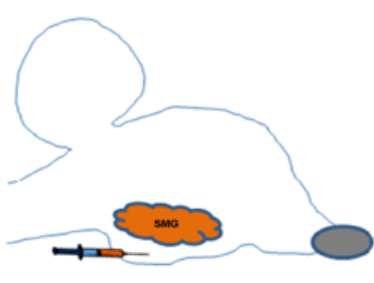

A cell transplantation model: HSG only or HSG and DPSC mixed with hyaluronic acid (HA) hydrogel were subcutaneously transplanted ventrally to the submandibular salivary gland (SMG) without penetrating in the gland in Rag1-/- mice ( $n=3$ per group) to avoid immune rejection against human cells (HSG).

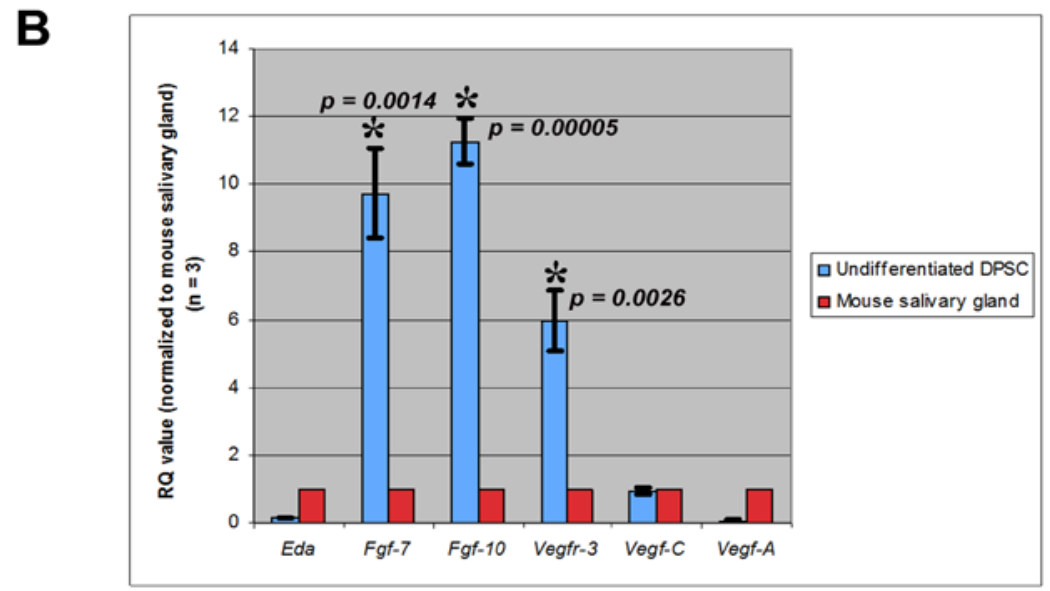

Figure 2: Cultured undifferentiated DPSC expressed high level of Fgf-7 and Fgf-10, neural crest-derived mesenchymal genes involving salivary gland formation. (A) A diagram illustrates the in vivo cell transplantation model. HSG alone or the combination between HSG and DPSC (1 million per cell type) were prepared as cell suspension in hyaluronic acid (HA) hydrogel scaffold and subcutaneously injected ventrally to the submandibular salivary gland (SMG) without penetrating in the gland in 2-month-old Rag1-/- null mice ( $n=3$ mice/group) to avoid immune rejection against the human cells (HSG). (B) Q-RT-PCR showed RQ (Relative Quantification) values demonstrating differential gene expression of Eda, Fgf-7, Fgf-10, Vegfr-3, Vegf-C, and Vegf-A in undifferentiated DPSC and mouse salivary gland. DPSC cultured in stem cell media under $5 \% \mathrm{O}_{2}$ incubation, as previously described in Materials and Methods, expressed high level of Fgf-7, Fgf-10, and Vegfr-3, as well as the same level of Vegf-C, compared to mouse salivary gland (approximately $>10$ folds greater than endogenous Fgf- 7 and Fgf-10, and $>5$ folds greater than endogenous Vegfr-3 expression in mouse submandibular salivary gland. $R Q$ values $(n=3)$ were normalized by the expression of mouse salivary gland. Gapdh was used for the internal control. Student's t-test calculated * $p \leq 0.05$. Error bars represent \pm SEM. 

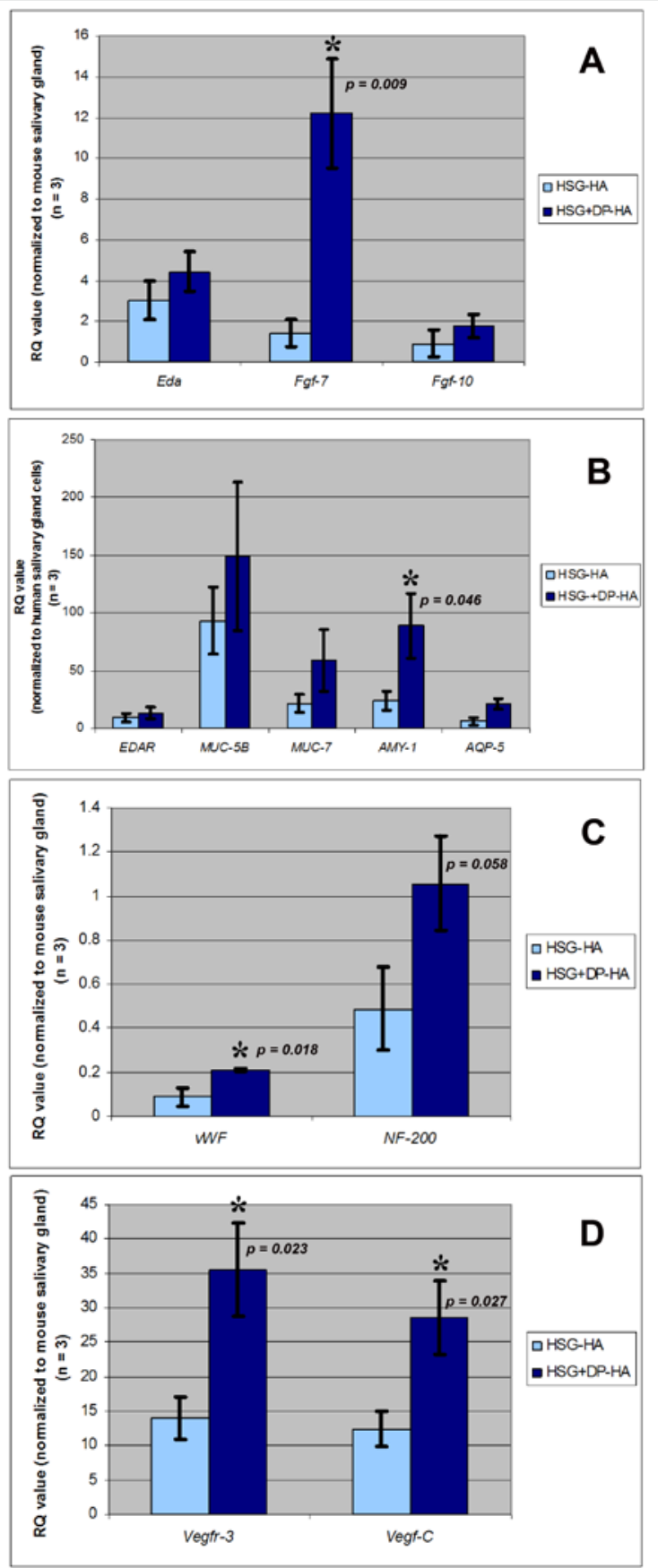

Figure 3: Co-transplantation of HSG and DPSC demonstrated high expression of murine neural crest-derived mesenchymal and human salivary gland genes. (A-D) Q-RT-PCR showed RQ (Relative Quantification) values demonstrating differential gene expression in HSG alone and HSG cotransplanted with DPSC. The transplanted HSG and DPSC in HA hydrogel scaffolds expressed higher levels of murine Eda, Fgf-7, and Fgf-10, human EDAR, MUC-5B, MUC-7, AMY-1, and AQP-5, as well as murine vWF, NF200, Vegfr-3, and Vegf-C. (A) HSG+DPSC particularly expressed significantly higher Fgf-7 expression (> 10 folds compared to HSG transplants alone). (B) The HSG alone and HSG co-transplanted with DPSC expressed higher level of EDAR, MUC-5B, MUC-7, AMY-1, AQP-5 (approximately >10-150 folds compared to undifferentiated human submandibular salivary gland cells). AMY1 was expressed significantly higher in the co-transplanted tissues. ( $C$ and D) HSG+DPSC expressed higher level of mouse vWF, NF-200, Vegfr-3 and Vegf-C compared to HSG transplants alone. $R Q$ values $(n=3)$ were normalized by the expression of mouse salivary gland or human salivary gland cells. Mouse Gapdh or human GAPDH was used for the internal control. Student's t-test calculated ${ }^{*} p \leq 0.05$. Error bars represent \pm SEM. culture group formed large multi-cellular structures that resembled intact salivary glands with acinar- and duct-like structures (Figure 1L, arrowheads). Noticeably, co-culturing DPSC shown by positive $\mathrm{X}$-gal staining (in blue) were clustered near HSG-derived acinar-like structure (Figure1J, arrows).

The HSG-derived acinar structures in both HSG alone and HSG+DPSC were positive for CD44 (in red) and lysosome associated membrane protein-1 (LAMP-1) (in green), confirming the differentiation of HSG to acinar cells (Figures $1 \mathrm{~K}$ and L). CD44 and LAMP-1 are expressed by HSG and acinar salivary gland $[23,24,26]$. The acinar structures in the HSG+DPSC group were strongly positive for LAMP-1 when compared to those in HSG alone, suggesting higher maturity of acinar salivary gland structures. Additionally, the number of acinar-like structures quantified in the HSG+DPSC group was significantly larger than those in HSG alone $\left({ }^{*} p=0.002\right)$ (Figure $1 \mathrm{M})$. Taken these results together, this suggests that DPSC enhance the ability of HSG to differentiate to acinar- and duct-like phenotypes.

\section{DPSC expressed high level of Fgf-7 and Fgf-10, neural crest- derived mesenchymal genes essential for salivary gland formation}

To complement our in vitro study and to gain insight into DPSC's supportive role to induce HSG differentiation into functional salivary gland units in vivo, we transplanted HSG alone or the combination between HSG and DPSC subcutaneously by direct injection of cell suspension ventrally to the submandibular salivary gland without penetrating in the gland with hyaluronic acid (HA) hydrogel in Ragl null mice to avoid immune rejection against the human cells (HSG) (Figure 2A).

Undifferentiated DPSC in our culture condition showed the expression of ectodysplasin, Eda, and fibroblast growth factors, Fgf7 and Fgf-10. Eda, Fgf-7, and Fgf-10 are proteins secreted by neural crest-derived mesenchymal cells to induce branching morphogenesis during salivary gland development and formation [7,25,27]. DPSC expressed significantly high levels of Fgf-7 and Fgf-10, which is approximately $>10$ folds greater than endogenous Fgf- 7 and Fgf- 10 expression in mouse submandibular salivary gland $\left({ }^{*} \mathrm{p}=0.0014\right.$ and 0.00005 , respectively) (Figure $2 \mathrm{~B}$ ). The high expression of both growth factors combined with our previous study showing that DPSC are neural crest-derived led us to hypothesize that DPSC may be a good source of ectomesenchymal supportive of salivary gland formation and regeneration [14]. In addition, DPSC expressed high level of vascular endothelial growth factor receptor 3, Vegfr-3 ( $>5$ folds greater than endogenous Vegfr- $3,{ }^{*} p=0.0026$ ), and similar level of its ligand, Vegf-C but not Vegf-A, when compared with that of mouse salivary gland (Figure 2B). A previous study demonstrated that stimulation of blood vessel formation improved regeneration of submandibular salivary gland [28]. Therefore, the expression of angiogenic genes by DPSC suggests that their angiogenic potential may be beneficial for salivary gland formation.

Co-transplantation of HSG and DPSC demonstrated high expression of murine neural crest-derived mesenchymal and human salivary gland differentiation genes

After two-week post-transplantation, HA hydrogel plugs were processed for histological and Q-RT-PCR analyses using primers specific for human salivary gland differentiation genes and mouse specific mesenchymal genes. 
DPSC up-regulated the expression of Eda, Fgf-7, and Fgf-10 in the HA hydrogel plugs in vivo. The level of all three mesenchymal genes expressed in HSG+DPSC was also greater than that expressed in HSG alone. In particular, the levels of Fgf- 7 showed $>10$ folds greater than endogenous Fgf-7 expression in untransplanted mouse submandibular gland $\left({ }^{*} \mathrm{p}=0.009\right)$ (Figure $\left.3 \mathrm{~A}\right)$. To confirm the formation of functional salivary gland, we used human specific primers to determine the expression of salivary gland differentiation genes. The HSG alone and co-transplanted with DPSC expressed higher level of human ectodysplasin receptor (EDAR), mucin (MUC-5B and MUC-7), alpha-amylase-1 (AMY-1), and aquaporin-5 (AQP-5) (approximately $>10-150$ folds greater than the expression in undifferentiated human submandibular salivary gland cells) (Figure 3B). Importantly, alphaamylase- 1 which is an enzyme that is secreted by a functional salivary gland, was expressed significantly higher in the co-transplanted tissues $\left({ }^{*} \mathrm{p}=0.046\right)$, suggesting that HSG co-transplanted with DPSC formed more mature and functional salivary gland.

Next, we studied if DPSC enhance human salivary gland cells to differentiate and form functional salivary gland by induction of blood vessel and nerve innervating formation. To answer this question, we examined endothelial-specific, neuronal, and angiogenic gene expression by Q-RT-PCR. Accordingly, HSG+DPSC group showed higher level of the endothelial markers, von Willebrand Factor $(v W F)$ $\left({ }^{*} \mathrm{p}=0.018\right)$ as well as the neuronal marker, heavy neurofilament $(\mathrm{NF}-200)(\mathrm{p}=0.058)$, when comparing with HSG group (Figure $3 \mathrm{C})$.
Likewise, the HSG+DPSC co-transplanted tissues expressed higher levels of Vegfr-3 and Vegf- $C\left({ }^{*} \mathrm{p}=0.023\right.$ and 0.027 , respectively) (approximately $>30-35$ folds and $>2$ folds greater than expression in mouse submandibular salivary gland and in HSG transplants, respectively) (Figure 3D). The expression of these transcripts suggests that DPSC enhance the salivary gland cells to differentiate and form salivary gland tissue by induction of blood vessel and nerve innervating formation.

\section{Glandular structures were observed in the co-transplantation of HSG and DPSC}

In addition to gene expression, we determined the morphology of transplants in HSG alone and HSG+DPSC. H\&E staining revealed that HSG hydrogel plugs showed only immature cancer-like cells with large nuclei (Figures 4A-C). Conversely, duct- and acinar-like structures represented by their polarized nuclei were seen in the hydrogel plugs containing HSG and DPSC, specially at the periphery close to mesenchymal cells (Figures 4D-F) and vessels (Figure 4E, inset).

PAS staining distinguished acinar- from duct-like structures by revealing the formation of mucin/mucopolysaccharide containing cells. PAS-positive cells (in pink) were randomly found in the HSG transplants alone (Figures 4G-I). In contrast, several clusters of PASpositive acinar- like with some PAS-negative duct-like structures were present in the HSG+DPSC transplants (Figure 4J, inset).

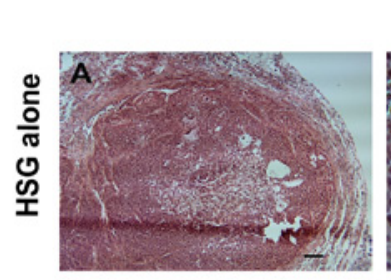

\section{H\&E staining}
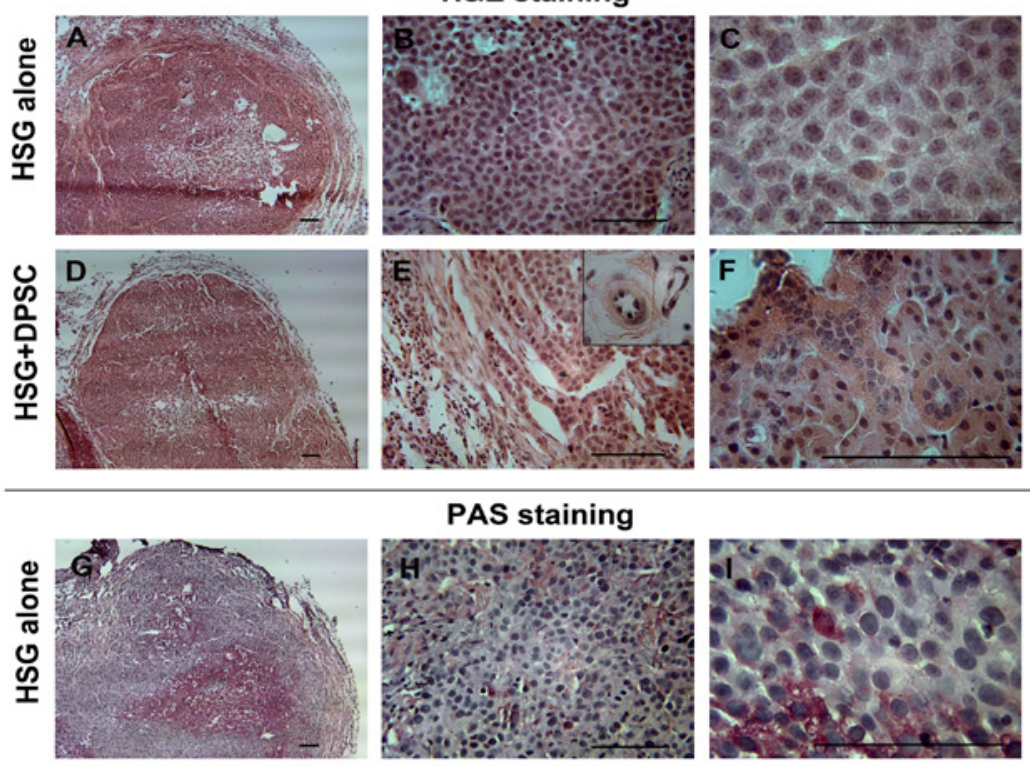

PAS staining
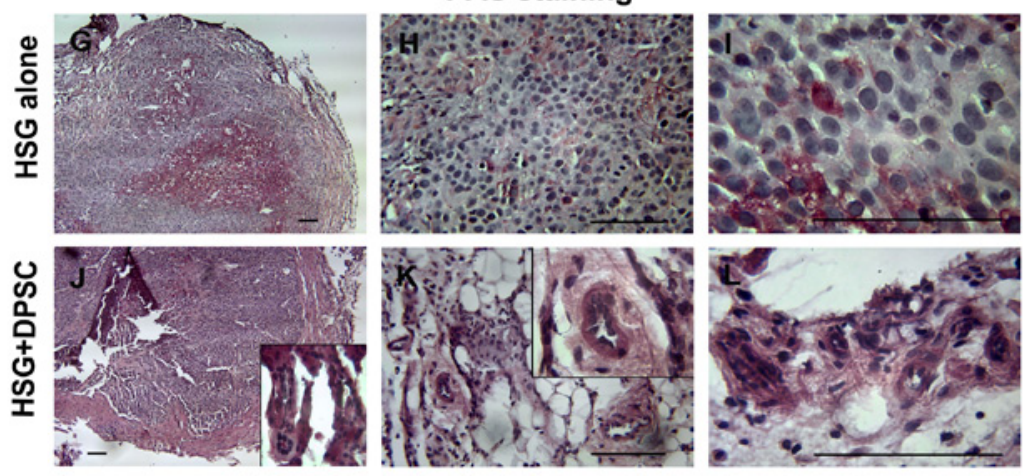

Figure 4: HSG co-transplanted DPSC demonstrated glandular structures with acinar- and duct-like structures. HSG only (1 million cells) or HSG and DPSC (1 million each) were injected in HA hydrogels subcutaneously ventrally to the submandibular salivary gland in Rag1 -/- mice ( $n=3$ mice per group). (A-C) H\&E staining demonstrated immature cancer-like cells with large nuclei in HSG hydrogel plugs. (D-F) H\&E staining showed the duct- and acinar-like structures represented by their polarized nuclei in the hydrogel plugs containing HSG and DPSC. (G-I) PAS staining indicated some randomly positive cells (in pink) were found in the HSG transplants alone. (J-L) Clusters of PAS-positive acinar-like with some PAS-negative duct- like structures were present in the HSG+DPSC transplants. (E and K, insets, and L) Blood vessels were found at the interface of HSG and DPSC where the glandular structures were observed. Scale bars indicate $100 \mu \mathrm{m}$. 

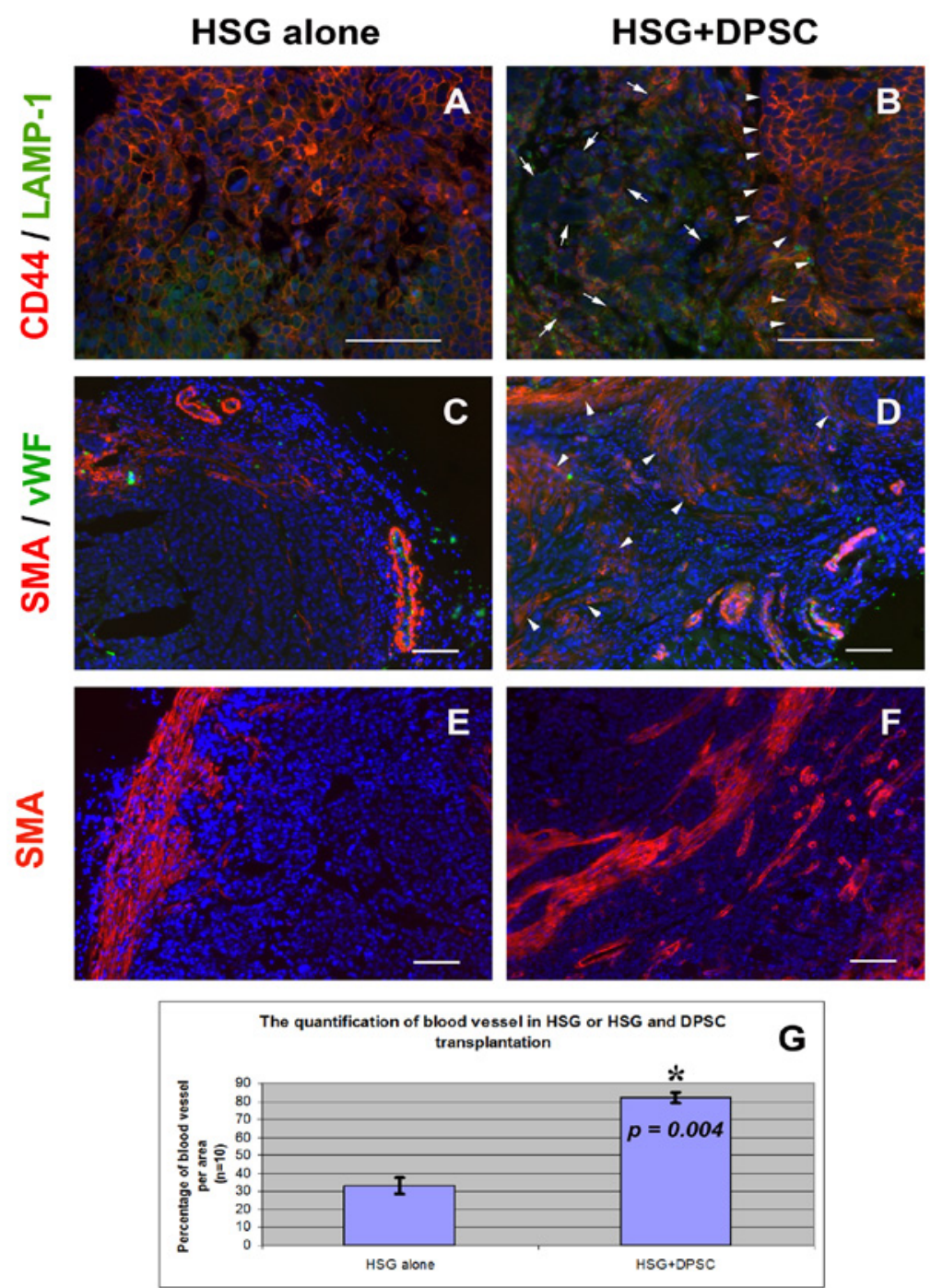

Figure 5: Immunohistological characterization of HSG and DPSC in HA hydrogels. (A and B) Mature glandular structures which were double-positive for CD44 and LAMP-1 (arrows) were seen in the HSG+DPSC co-transplantation group, but not in the HSG alone. (A) The cells in HSG transplants stained positive for CD44, but negative for LAMP-1, suggesting their immature state. (B, arrowheads) Differentiated HSG cells were observed at the interface with DPSC which encapsulated the HSG tumor. (C-F) Encapsulating DPSC which were positively stained for SMA (arrowheads) attempted to invade into the transplanted tissue and also recruited blood vessels. The blood vessels were recognized by SMA and vWF staining. (G) The bar graph shows the quantification of blood vessels as percentage per area in both HSG and HSG+DPSC transplants. The number of blood vessels in HSG+DPSC $(82 \% \pm 1.37)$ was significantly higher compared to that in HSG alone (33\% \pm 0.91$)$. The number of blood vessels in both transplants were measures from 10 areas of three samples per transplant $(n=10)$. Student's t-test calculated * $p \leq 0.05$. Error bars represent \pm SEM. Scale bars indicate $100 \mu \mathrm{m}$.

Immunofluorescence showed more mature glandular structures which were double- positive for CD44 and LAMP-1 in the HSG+DPSC co-transplantation group, but not in the HSG alone (Figures $5 \mathrm{~A}$ and $\mathrm{B}$, arrows). The cells in HSG transplants were immature which stained positive for CD44, but negative for LAMP-1 (Figure 5A). Interestingly, differentiated HSG cells were observed at the interface with mesenchymal cells (DPSC) which encapsulated the HSG tumor (Figure 5B, arrowheads). In addition, encapsulating DPSC which stained positive for smooth muscle actin (SMA) integrated deeper into the core of the transplanted tissue and also recruited blood vessels (Figures $4 \mathrm{E}$ and $\mathrm{K}$, insets, $4 \mathrm{~L}, 5 \mathrm{C}$ and $\mathrm{D}$, arrowheads, 5E and $\mathrm{F}$ ). The blood vessels were recognized by SMA and vWF staining (Figures $5 \mathrm{C}-\mathrm{F}$ ). The quantification of blood vessels in both transplanted tissues showed significantly increased number of blood vessels in HSG+DPSC than that in HSG alone $\left({ }^{*} \mathrm{p}=0.004\right.$ ) (Figure $5 \mathrm{G}$ ).

\section{Discussion}

In general stem cell therapy focuses on delivering only the desired stem cell population. This approach works if the stem cell can regenerate the whole organ. For salivary gland regeneration, although a putative salivary gland stem cell can potentially regenerate all the epithelial components it cannot give rise to the supportive tissue including mesenchyme, vasculature and nerves. Since dental pulp stem cells are neural crest-derived and epithelial- mesenchymal interactions of tooth bud and salivary glands are similar, we hypothesized that DPSC is a good source of mesenchyme to induce and support salivary gland tissue differentiation. 
We first studied the effects of co-culture with DPSC on HSG differentiation in vitro. DPSC and HSG were co-cultured on Matrigel. After 4 days, HSG had formed acini but the number of acini and the size of the acini was significantly increased in the co-cultures with HSG as compared to HSG only cultures.

Matrigel's unknown composition and immunogenicity are some of the disadvantages for its in vivo use [29,30]. Thus we sought a more natural matrix for salivary gland formation. Hyaluronic acid (HA) is the most abundant glycosaminoglycan in the developing salivary gland [22]. Also, adult salivary gland secretory units produce high amount of HA, which is deposited in the basal lamina [21]. Furthermore, acinar epithelial cells express CD44 the receptor for HA [31,32]. Thus we hypothesized that HA hydrogels will provide a good a natural scaffold for salivary gland formation and epithelial-mesechymal interaction. Therefore, we conducted xenotransplantation of HSG alone or HSG and DPSC in HA hydrogels subcutaneously ventrally to the endogenous submandibular gland. Although HA hydrogels have been previously used to induced 3D formation of salivary secretory units in vitro [33], to our knowledge this is the first report that demonstrate the potential use of HA hydrogels for in vivo formation of salivary gland tissue.

We performed transplantation of DPSC and HSG in Rag1 null mice. Using this hetero- xenotransplantation approach, we can clearly monitor and distinguish the contribution of murine DPSC using murine specific mesenchymal primers compared to untransplanted murine salivary gland tissue as well as the contribution and differentiation stage of HSG using human specific primers of salivary gland epithelial markers. Before transplantation, we observed that DPSC expressed approximately 10 folds higher levels of Fgf-7 and Fgf- 10 . These fibroblast growth factors are essential for proper salivary gland formation as their respective knockout and their receptor Fgfr-1 knockout mouse models result in salivary gland development defects or aplasia $[7,34]$. Furthermore, upon transplantation, DPSC expressed significantly higher levels of Fgf-7 as compared to untransplanted murine salivary gland and HGS only transplants. This is therapeutically significant as FGF-7 (aka Keratinocyte Growth Factor, KGF) administration has been shown beneficial for salivary gland restoration $[35,36]$.

Given the tumorigenic nature of HSG, we only performed short term transplantation. Nonetheless, in 2 weeks post-transplantation we showed significant increase in the levels of human salivary gland differentiation markers in both HSG only and HSG with DPSC cotransplantations. Moreover, gene expression of human alpha-amylase-1 (AMY-1) was significantly increased in the HSG co-transplanted with DPSC indicating that DPSC induced functional differentiation of HSG in vivo. Upon histological examination it became obvious that differentiated glandular structures were observed at the interface with mesenchyme and near vessels. This underlies the importance of epithelial-mesenchymal interaction for proper glandular differentiation and demonstrates that DPSC is a good source of ecto-mesenchyme. Consistent with our previous observations, DPSC exhibit great angiogenic capacity in vivo [14]. DPSC express high levels of Vegfr-3 and Vegf-C and significant higher levels of these angiogenic factors were found in the co-transplanted tissue containing DPSC and HSG as compared to HSG alone. This may explain the increased number of vessels in the co- transplanted tissue. This suggests that angiogenesis may be an important aspect of salivary gland tissue development and regeneration. Furthermore, we observed near significant higher levels of NF-200 that reached the levels seen in normal submandibular gland. Recently it has been demonstrated that innervation is crucial for normal development of the salivary gland [37]. Future studies are warranted to understand the mechanisms of DPSC induction of angiogenesis and neurogenesis.

In summary we provide evidence of the potential use of DPSC as ecto-mesenchyme for induction and support of salivary gland development. DPSC is an easily accessible stem cell source from third molars and their multi-lineage stem cell differentiation capacity combined with their trophic epithelial-morphogenic, angiogenic and neurogenic capacity makes then an ideal inductive and supportive cell source for salivary gland tissue engineering approaches.

\section{Acknowledgements}

We will like to thank Dr. Deok-Ho Kim for providing the HA hydrogels and Dr. Ken Isutzu for providing the HSG cell line. Funding for this research has been provided by the Departments of Pathology and Lab Medicine at the University of Washington.

\section{References}

1. Dirix P, Nuyts S, Vander Poorten V, Delaere P, Van den Bogaert W (2008) The influence of xerostomia after radiotherapy on quality of life: results of a questionnaire in head and neck cancer. Support Care Cancer 16: 171-179.

2. Lombaert IM, Brunsting JF, Wierenga PK, Faber H, Stokman MA, et al. (2008) Rescue of salivary gland function after stem cell transplantation in irradiated glands. PLoS One 3: e2063.

3. Bücheler M, Haisch A (2003) Tissue engineering in otorhinolaryngology. DNA Cell Biol 22: 549-564.

4. Yamamoto S, Fukumoto E, Yoshizaki K, Iwamoto T, Yamada A, et al. (2008) Platelet-derived growth factor receptor regulates salivary gland morphogenesis via fibroblast growth factor expression. J Biol Chem 283: 23139-23149.

5. Jaskoll T, Zhou YM, Chai Y, Makarenkova HP, Collinson JM, et al. (2002) Embryonic submandibular gland morphogenesis: stage-specific protein localization of FGFs, BMPs, Pax6 and Pax9 in normal mice and abnormal SMG phenotypes in FgfR2-IIIc(+/Delta), BMP7(-/-) and Pax6(-/-) mice. Cells Tissues Organs 170: 83-98.

6. Patel VN, Rebustini IT, Hoffman MP (2006) Salivary gland branching morphogenesis. Differentiation 74: 349-364.

7. Jaskoll T, Abichaker G, Witcher D, Sala FG, Bellusci S, et al. (2005) FGF10/ FGFR2b signaling plays essential roles during in vivo embryonic submandibular salivary gland morphogenesis. BMC Dev Biol 5: 11.

8. Madan AK, Kramer B; 58, 60-1 (2005) Immunolocalization of fibroblast growth factor-2 (FGF-2) during embryonic development of the rat submandibular gland. SADJ 60: 58, 60-61.

9. Jaskoll T, Witcher D, Toreno L, Bringas P, Moon AM, et al. (2004) FGF8 dose-dependent regulation of embryonic submandibular salivary gland morphogenesis. Dev Biol 268: 457-469.

10. Hoffman MP, Kidder BL, Steinberg ZL, Lakhani S, Ho S, et al. (2002) Gene expression profiles of mouse submandibular gland development: FGFR1 regulates branching morphogenesis in vitro through BMP- and FGF-dependent mechanisms. Development 129: 5767-5778.

11. Jaskoll T, Leo T, Witcher D, Ormestad M, Astorga J, et al. (2004) Sonic hedgehog signaling plays an essential role during embryonic salivary gland epithelial branching morphogenesis. Dev Dyn 229: 722-732.

12. Hai B, Yang Z, Millar SE, Choi YS, Taketo MM, et al. (2010) Wnt/ß-catenin signaling regulates postnatal development and regeneration of the salivary gland. Stem Cells Dev 19: 1793-1801.

13. Dang H, Lin AL, Zhang B, Zhang HM, Katz MS, et al. (2009) Role for Notch signaling in salivary acinar cell growth and differentiation. Dev Dyn 238: $724-$ 731.

14. Janebodin K, Horst OV, leronimakis N, Balasundaram G, Reesukumal K, et al. 
Citation: Janebodin K, Reyes M (2012) Neural Crest-Derived Dental Pulp Stem Cells Function as Ectomesenchyme to Support Salivary Gland Tissue Formation. Dentistry S13:001. doi:10.4172/2161-1122.S13-001

(2011) Isolation and characterization of neural crest-derived stem cells from dental pulp of neonatal mice. PLoS One 6: e27526.

15. Hayashi Y, Yanagawa T, Yoshida H, Yura Y, Nitta T, et al. (1985) Induction of other differentiation stages in neoplastic epithelial duct and myoepithelial cells from the human salivary gland grown in athymic nude mice. Cancer 55: 2575-2583.

16. Sato M, Hayashi Y, Yanagawa T, Yoshida H, Yura Y, et al. (1985) Intermediatesized filaments and specific markers in a human salivary gland adenocarcinoma cell line and its nude mouse tumors. Cancer Res 45: 3878-3890.

17. Maria OM, Maria O, Liu Y, Komarova SV, Tran SD (2011) Matrigel improves functional properties of human submandibular salivary gland cell line. Int $J$ Biochem Cell Biol 43: 622-631.

18. Shirasuna K, Sato M, Miyazaki T (1981) A neoplastic epithelial duct cell line established from an irradiated human salivary gland. Cancer 48: 745-752.

19. Vag J, Byrne EM, Hughes DH, Hoffman M, Ambudkar I, et al. (2007) Morphological and functional differentiation of HSG cells: role of extracellular matrix and trpc 1. J Cell Physiol 212: 416-423.

20. Cheng N, Lambert DL (2011) Mammary Transplantation of Stromal Cells and Carcinoma Cells in C57BL/6J Mice. J Vis Exp.

21. Cutler LS, Christian CP, Rendell JK (1987) Glycosaminoglycan synthesis by adult rat submandibular salivary-gland secretory units. Arch Oral Biol 32: 413419.

22. Cohn RH, Banerjee SD, Bernfield MR (1977) Basal lamina of embryonic salivary epithelia. Nature of glycosaminoglycan and organization of extracellular materials. The Cell Biol 73: 464-478.

23. Maria O, Maria A, Cai Y, Tran S (2012) Cell surface markers CD44 and CD166 localized specific populations of salivary acinar cells. Oral Dis 18: 162-168.

24. Fonseca I, Moura Nunes JF, Soares J (2000) Expression of CD44 isoforms in normal salivary gland tissue: an immunohistochemical and ultrastructural study. Histochem Cell Biol 114: 483-488.

25. Tucker AS (2007) Salivary gland development. Semin Cell Dev Biol 18: 237244.
26. Heffernan M, Yousefi S, Dennis JW (1989) Molecular characterization of P2B/ LAMP-1, a major protein target of a metastasis-associated oligosaccharide structure. Cancer Res 49: 6077-6084.

27. Morita K, Nogawa H (1999) EGF-dependent lobule formation and FGF7dependent stalk elongation in branching morphogenesis of mouse salivary epithelium in vitro. Dev Dyn 215: 148-154.

28. Lombaert IM, Brunsting JF, Wierenga PK, Kampinga HH, de Haan G, et al (2088) Cytokine treatment improves parenchymal and vascular damage of salivary glands after irradiation. Clin Cancer Res 14: 7741-7750.

29. Kilarski W, Bikfalvi A (2007) [Experimental approaches to study in vivo angiogenesis]. Bull Cancer 94 Spec No: S166-169.

30. Norrby K (2006) In vivo models of angiogenesis. J Cell Mol Med 10: 588-612.

31. Xing R, Regezi JA, Stern M, Shuster S, Stern R (1998) Hyaluronan and CD44 expression in minor salivary gland tumors. Oral Dis 4: 241-247.

32. Terpe HJ, Stark H, Prehm P, Günthert U (1994) CD44 variant isoforms are preferentially expressed in basal epithelial of non-malignant human fetal and adult tissues. Histochemistry 101: 79-89.

33. Pradhan S, Liu C, Zhang C, Jia X, Farach-Carson MC, et al. (2010) Lumen formation in three-dimensional cultures of salivary acinar cells. Otolaryngol Head Neck Surg 142: 191-195.

34. Makarenkova HP, Hoffman MP, Beenken A, Eliseenkova AV, Meech R, et al. (2009) Differential interactions of FGFs with heparan sulfate control gradient formation and branching morphogenesis. Sci Signal 2: ra55.

35. Lombaert IM, Brunsting JF, Wierenga PK, Kampinga HH, de Haan G, et al (2008) Keratinocyte growth factor prevents radiation damage to salivary glands by expansion of the stem/progenitor pool. Stem Cells 26: 2595-2601.

36. Zheng C, Cotrim AP, Rowzee A, Swaim W, Sowers A, et al. (2011) Prevention of radiation-induced salivary hypofunction following hKGF gene delivery to murine submandibular glands. Clin Cancer Res 17: 2842-2851.

37. Knox SM, Lombaert IM, Reed X, Vitale-Cross L, Gutkind JS, et al. (2010) Parasympathetic innervation maintains epithelial progenitor cells during salivary organogenesis. Science 329: 1645-1647. 